\title{
Critical Behavior at the Chiral Phase Transition *
}

C. Bernard ${ }^{\mathrm{a}}$, T. Blum ${ }^{\mathrm{b}}$, C.E. DeTar ${ }^{\mathrm{c}}$, Steven Gottlieb ${ }^{\mathrm{d}}$, U.M. Heller ${ }^{\mathrm{e}}$, J.E. Hetrick ${ }^{\mathrm{a}}$, Beat Jegerlehner ${ }^{\mathrm{d}}$, K. Rummukainen ${ }^{\mathrm{f}}$, R.L. Sugar ${ }^{\mathrm{g}}$, D. Toussaint ${ }^{\mathrm{h}}$, and M. Wingate ${ }^{\mathrm{i}}$,

${ }^{a}$ Department of Physics, Washington University, St. Louis, MO 63130, USA

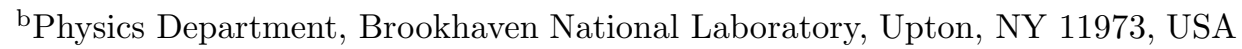

${ }^{\mathrm{c}}$ Physics Department, University of Utah, Salt Lake City, UT 84112, USA

and Zentrum für interdisziplinäre Forschung, Universität Bielefeld, Bielefeld, Germany

${ }^{\mathrm{d}}$ Department of Physics, Indiana University, Bloomington, IN 47405, USA

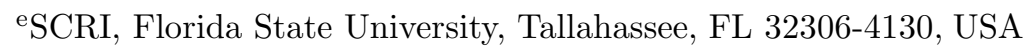

${ }^{\mathrm{f}}$ Fakultät für Physik, Universität Bielefeld, Bielefeld, Germany

${ }^{\text {g} D e p a r t m e n t ~ o f ~ P h y s i c s, ~ U n i v e r s i t y ~ o f ~ C a l i f o r n i a, ~ S a n t a ~ B a r b a r a, ~ C A ~ 93106, ~ U S A ~}$

${ }^{\mathrm{h}}$ Department of Physics, University of Arizona, Tucson, AZ 85721, USA

and Center for Computational Physics, University of Tsukuba, Ibaraki 305, Japan

${ }^{\mathrm{i} D}$ Department of Physics, University of Colorado, Boulder, CO 80309, USA

\section{INTRODUCTION}

It is generally expected that two-flavor QCD undergoes a high temperature chiral symmetry restoring phase transition at zero quark mass, with $\mathrm{O}(4)$ critical behavior [1]. Verifying this expectation is important for understanding the phenomenology of the transition and for facilitating an extrapolation of simulation data to physical quark masses. Lattice QCD at fixed $N_{t}$ with staggered fermions is similarly expected to exhibit at least $\mathrm{O}(2)$ universality, with $\mathrm{O}(4)$ emerging in the continuum limit. For present purposes we do not distinguish $\mathrm{O}(2), \mathrm{O}(4)$, or mean field critical behavior.

One test of universality compares critical exponents. Comparing the critical scaling function gives further insight. We use the standard correspondence between $\mathrm{QCD}$ variables and $\mathrm{O}(\mathrm{N})$ spin

*Presented by C. DeTar. Supported by the US DOE and NSF. Computations were done at SDSC, NCSA, PSC, Cornell CTC, and on the UCSB Origin 2000. variables, which identifies quark mass $m_{q} / T$ with magnetic field $h$, inverse gauge coupling $6 / g^{2}$ with temperature $T / T_{c}(0)$, and chiral condensate $\bar{\psi} \psi$ with magnetization $M$. A critical point is expected to occur at zero quark mass and nonzero coupling $6 / g^{2}(0)$. For studies at fixed $N_{t}$ we use[2]

$$
\begin{aligned}
h & =a m_{q} N_{t} \\
t & =T / T_{c}(0)-1=6 / g^{2}-6 / g^{2}(0)
\end{aligned}
$$

Critical scaling theory predicts that for small quark masses we have the scaling relation [3]

$\bar{\psi} \psi h^{-1 / \delta}=f_{Q C D}(x)=c_{y} f_{O(4)}\left(c_{x} x\right)$

where $x=t h^{-1 / \beta \delta}, c_{x}$ and $c_{y}$ are scale constants, $f_{Q C D}(x)$ is the critical scaling function for QCD and $f_{O(4)}(x)$ is that for $\mathrm{O}(4)$. Not shown are nonleading nonscaling contributions analytic in $t$ and $h$.

\section{A CRITICAL EXPONENT}

We extended an old data sample at $N_{t}=4$, which was done with the standard one-plaquette 
gauge action plus two-flavor staggered fermion action. Our new sample decreases the quark mass to $a m_{q}=0.008$ and increases the spatial lattice size $L$ to 24 . We also reanalyze old data at $N_{t}=6,8,12$ 画. The latter all have $L=2 N_{t}$.

The mixed plaquette/chiral condensate susceptibility

$$
\chi_{m t}=\partial(\bar{\psi} \psi) / \partial\left(6 / g^{2}\right)
$$

has a peak, which can be used to locate the crossover. We extrapolate the peak height to infinite volume as shown below.

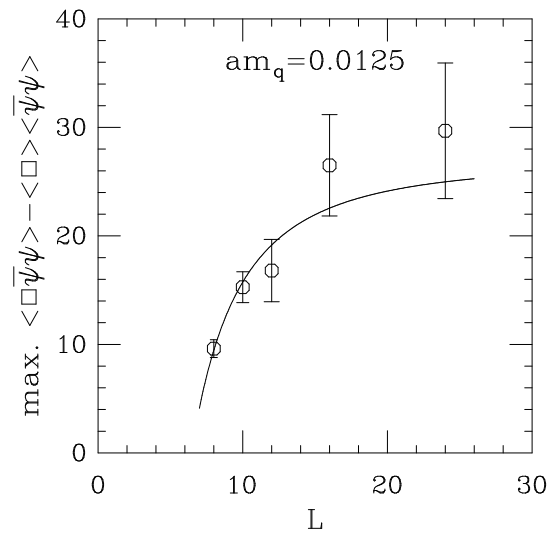

The infinite volume value should scale as

$$
\chi_{m t, \max } \sim\left(a m_{q}\right)^{(\beta-1) / \beta \delta} \approx\left(a m_{q}\right)^{-0.33}
$$

The figure below shows a strong departure from scaling at present quark masses, in agreement with similar findings of the JLQCD and Bielefeld groups for a variety of susceptibilities 5 .

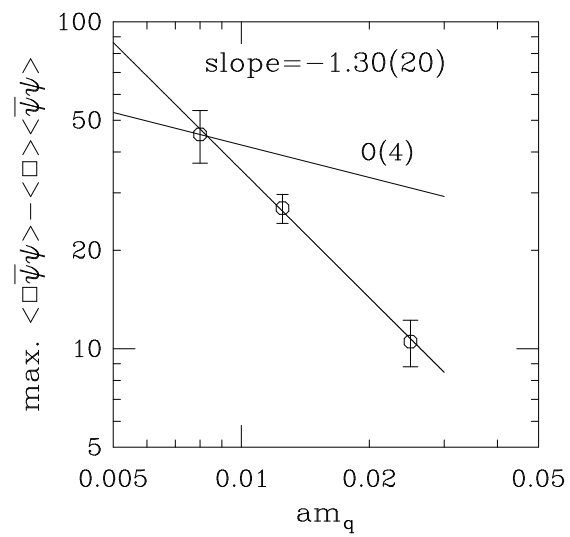

\section{SCALING FUNCTION AT $N_{t}=4$}

To find the critical coupling at zero quark mass, $6 / g^{2}(0)$, we use the scaling relation for the crossover (pseudocritical) coupling [2]:

$$
6 / g_{p c}^{2}=6 / g^{2}(0)+x_{p c}\left(N_{t} a m_{q}\right)^{1 / \beta \delta}
$$

which works well.

Neglecting nonscaling contributions to $\bar{\psi} \psi$, we construct the QCD critical scaling function (for $x>0$ ) using $\mathrm{O}(4)$ critical exponents $[6]$ and compare with the scaling function for $\mathrm{O}(4)$ [7] in the figure below. Vertical and horizontal displacements of the log-log scaling curves are permitted and correspond to adjusting $c_{x}$ and $c_{y}$.

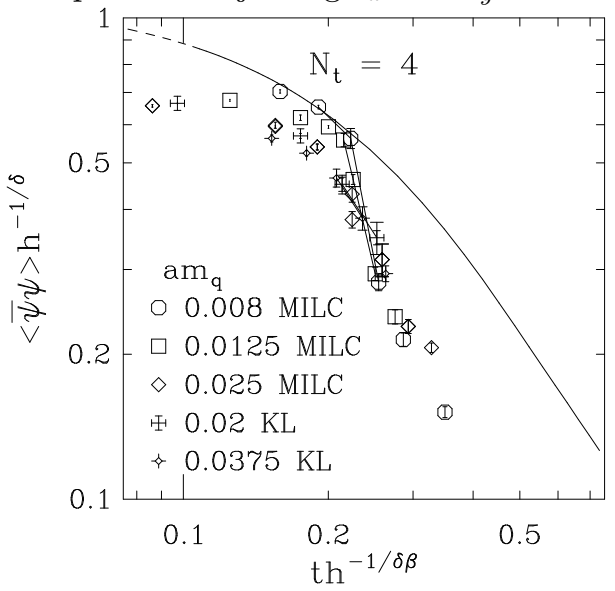

The line segments locate the crossover in the QCD data and the dashed line on the $\mathrm{O}(4)$ curve, the crossover in $\mathrm{O}(4)$. The newer data are plotted with octagons and squares.

We see: (1) The new data show generally worse agreement with the $\mathrm{O}(4)$ scaling curve. (2) In terms of these scaling variables and at these quark masses, the $\mathrm{O}(4)$ critical region is quite small, if it is to be found at all. Equivalently, nonnegligible nonscaling contributions may be masking critical behavior in this regime. (3) Nonscaling contributions could be displacing the crossover location.

\section{LARGER $N_{t}$}

A similar analysis at larger $N_{t}$ is shown below. Perhaps there is improvement with increasing $N_{t}$. However, our $N_{t}=12$ sample includes data only 
at a single quark mass, making it our weakest example. Furthermore, for $N_{t}>4$ we have no results for $L>2 N_{t}$.
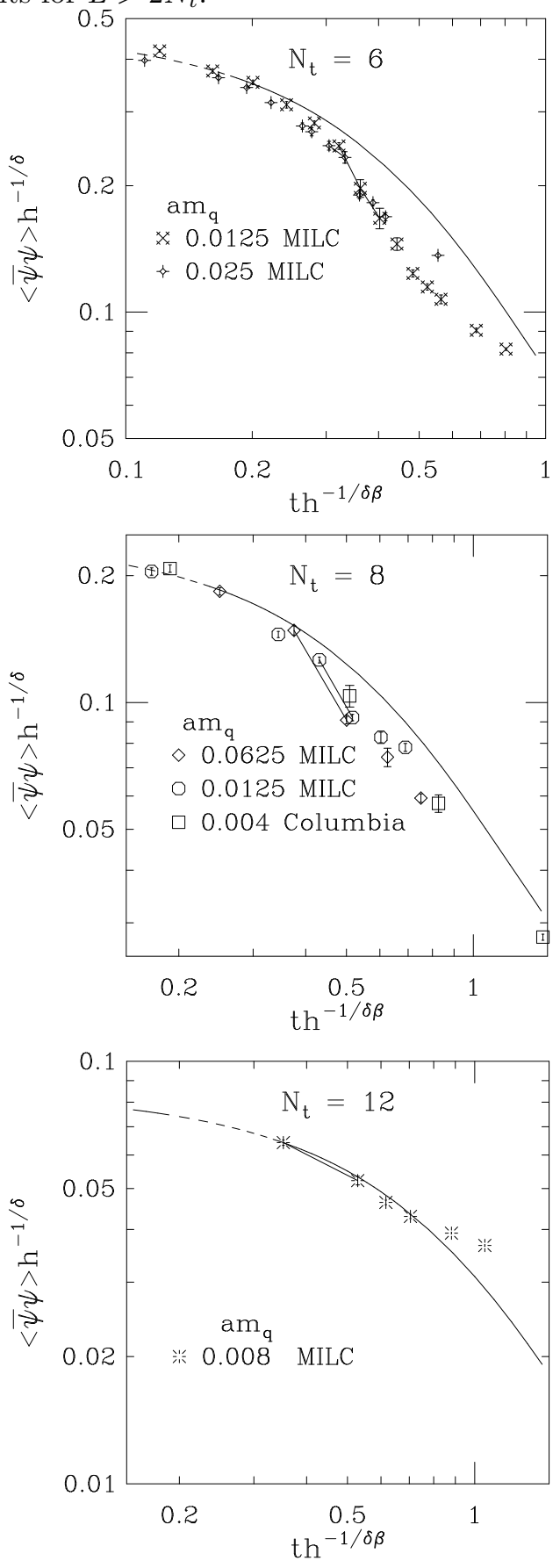

\section{SPECULATIONS}

New results at smaller quark mass and larger volume at $N_{t}=4$ have raised doubts about the extent of the previously observed agreement between QCD and $\mathrm{O}(4)$ 2, 2, A]. In striking contrast with Wilson fermions with an improved gauge action [8], the conventional staggered fermion action with the conventional choice of scaling variables does not show good agreement with the $\mathrm{O}(4)$ scaling function at present quark masses and temperatures.

Why? (1) Perhaps the critical region is attained only when $\pi$ and $\sigma$ correlation lengths are considerably greater than $1 / T_{c}$. Here, typically, they are smaller. (2) At strong coupling the variables $6 / g^{2}$ and $m a$ may have a strongly nonlinear correspondence with spin model temperature and magnetic field $T, h$, which becomes approximately linear only in a small region around the critical point. The nonlinearities may become weaker in the weak coupling limit and perhaps may be diminished with improved actions. Perhaps with a more suitable choice of scaling variables, we may find better agreement.

\section{REFERENCES}

1. R. Pisarski and F. Wilczek, Phys. Rev. D 29 (1984) 338.

2. F. Karsch and E. Laermann, Phys. Rev. D 50 (1994) 6954.

3. D.J. Amit, Field Theory, the Renomalization Group, and Critical Phenomena, (McGraw Hill, New York, 1978).

4. C. Bernard et al, Phys. Rev. D 54 (1996) 4585.

5. A. Ukawa, Lattice '96, Nucl. Phys. B (PS) 53(1997) 95.

6. K. Kanaya and S. Kaya, Phys. Rev. D 51 (1995) 2404.

7. D. Toussaint, Phys. Rev. D 55, 362 (1997).

8. Y. Iwasaki, K. Kanaya, S. Kaya, T. Yoshie, Phys. Rev. Lett. 78 (1997) 179 and S. Kaya, this conference. 